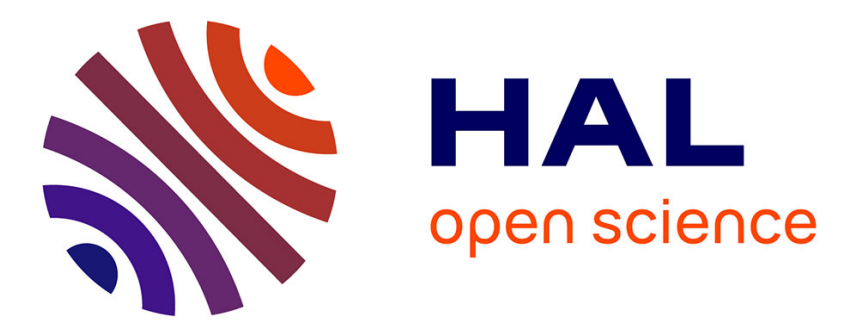

\title{
Improvement of motion estimation by assessing the errors on the evolution equation
}

\author{
Isabelle Herlin, Dominique Béréziat, Nicolas Mercier
}

\section{To cite this version:}

Isabelle Herlin, Dominique Béréziat, Nicolas Mercier. Improvement of motion estimation by assessing the errors on the evolution equation. VISAPP 2012: International Conference on Computer Vision Theory and Applications, Feb 2012, Rome, Italy. pp.235-240. hal-00677662

\section{HAL Id: hal-00677662 \\ https://hal.inria.fr/hal-00677662}

Submitted on 14 Mar 2012

HAL is a multi-disciplinary open access archive for the deposit and dissemination of scientific research documents, whether they are published or not. The documents may come from teaching and research institutions in France or abroad, or from public or private research centers.
L'archive ouverte pluridisciplinaire HAL, est destinée au dépôt et à la diffusion de documents scientifiques de niveau recherche, publiés ou non, émanant des établissements d'enseignement et de recherche français ou étrangers, des laboratoires publics ou privés. 


\title{
Improvement of motion estimation by assessing the errors on the evolution equation
}

\author{
Isabelle Herlin ${ }^{1,2}$ and Dominique Bereziat ${ }^{3}$ and Nicolas Mercier ${ }^{1,2}$ \\ ${ }^{1}$ INRIA, Institut National de Recherche en Informatique et Automatique, Domaine de Voluceau, Rocquencourt - BP 105, \\ 78153 Le Chesnay Cedex, France \\ ${ }^{2}$ CEREA, joint laboratory ENPC - EDF R\&D, Université Paris-Est, 6-8 avenue Blaise Pascal, Cité Descartes, \\ Champs-sur-Marne, 77455 Marne la Vallée Cedex 2, France \\ ${ }^{3}$ UPMC, Université Pierre et Marie Curie, 4 place Jussieu 75005 Paris, France \\ Isabelle.Herlin@inria.fr,Dominique.Bereziat@lip6.fr
}

Keywords: Optical Flow; Data Assimilation; Optimal Control

\begin{abstract}
Image assimilation methods are nowadays widely used to retrieve motion from image sequences with heuristics on the underlying dynamics. A mathematical model on the temporal evolution of the motion field has to be chosen, according to these heuristics, that approximately describes the evolution of the velocity at a pixel over the sequence. In order to quantify this approximation, we add an error term in the evolution equation of the motion field and design a weak formulation of 4D-Var image assimilation. The designed cost function simultaneously depends on the initial motion field and on the error value at each time step. The BFGS solver performs minimization to retrieve both motion field and errors. The method is evaluated and quantified on twin experiments, as no ground truth would be available for real data. The results demonstrate that the motion field is better estimated thanks to the error control.
\end{abstract}

\section{INTRODUCTION}

Motion estimation from an image sequence is one major issue of Image Processing in a large range of applicative domains. This allows for instance to study the dynamics of clouds and estimate the ocean surface circulation on satellite data. The retrieved motion fields can be further used as pseudo-observations for 3D models.

In the Image Processing literature, motion fields are most often inferred from an equation describing the transport of pixel brightness by velocity (for instance the optical flow constraint equation used in (Horn and Schunk, 1981) and (Isambert et al., 2008), or the mass conservation equation of (Béréziat et al., 2000). By nature, this transport equation is under-constrained to retrieve the two components of motion on 2D image data and spatial regularization techniques are used to define a well-posed problem with a unique solution. This is the well-known Tikhonov regularization, (Tikhonov, 1963).

However, heuristics on the dynamics displayed by the image sequence are often available and should be used to solve the problem of retrieving motion fields from image data. The first concern is to select the mathematical equations that optimally describe that dynamics. The second one is to define the process of motion estimation from these equations and from the links between the evolution of image values and the motion field. Variational data assimilation methods, and in particular 4D-Var methods, are emerging techniques in the Image Processing community that allow this retrieval of motion from images, (Papadakis et al., 2007), (Titaud et al., 2010) and (Béréziat and Herlin, 2011)). These 4D-Var methods solve a system of three equations that describes the temporal evolution of the state vector, the links between the observation values and state vector, and the background value of the state vector. The name "background" comes from the data assimilation community and refers to the value given to the state vector at the beginning of the optimization process. The solution of this system of equations is then formulated as the solution of an optimization problem.

The first 4D-Var method, (Le Dimet and Talagrand, 1986), supposes that the state vector evolution equation has no error and perfectly describes the dynamics: the model is a Perfect Model (PM). In that case, the control variable is reduced to the initial value of the state vector at the beginning of the studied tem- 
poral window, named assimilation window. The data assimilation method is named strong 4D-Var.

Unfortunately, the heuristics that are available on the dynamics of an image sequence are only approximating the reality. It is then valuable and wise to assess that approximation by introducing an error in the evolution equation as in (Trémolet, 2006). A weak formulation of 4D-Var, which includes such error term $\varepsilon(t)$ in the evolution equation, is extensively described in (Valur Hólm, 2008). It is used to solve image processing problems such as motion estimation, (Béréziat and Herlin, 2011), or curve tracking, (Papadakis et al., 2005). The control variable of the optimization problem becomes the state vector value over the whole assimilation window. The incremental method used to solve the optimization in (Valur Hólm, 2008) has however a major drawback: it has a slow convergence compared to the steepest gradient methods used for strong 4D-Var methods. An alternative is to control the initial value of the state vector and the error terms. The advantage is that it allows an efficient minimization by the same methods used for perfect models. This is applied for instance in (Papadakis et al., 2007), even if authors do not discuss the impact on the results of including that error term.

This paper analyses the advantages of adding an error term $\varepsilon(t)$ in the state vector dynamic equation for the issue of motion estimation by image assimilation and quantifies the impact on results. In Section 2 we define the state vector and the system of equations used for image assimilation and motion estimation. We introduce a pseudo-image component in the state vector and justify its interest for image assimilation. Section 3 describes the 4D-Var method that simultaneously controls the initial condition and the error term $\varepsilon(t)$ added to the evolution equation. Details on the computation of the gradient of the cost function are given in that section. Section 4 provides information about the numerical implementation to allow duplication of the method by interested Readers. Twin experiments are designed in Section 5 in order to evaluate quantitatively the impact of the error term on the quality of estimated motion fields. This is the only way to obtain a ground truth of the error involved in the evolution equation. We last conclude in Section 6 .

\section{Mathematical settings}

Let $\Omega$, a rectangle of $\mathbb{R}^{2}$, denote the bounded image domain, $\left[t_{0}, t_{N}\right]$ denote the temporal assimilation window and define $A=\Omega \times\left[t_{0}, t_{N}\right]$ the space-time domain. We also denote $H(\Omega)$ the Sobolev space included in $L^{2}(\Omega)$, space of square-integrable function from $\Omega$ to $\mathbb{R}$. A location $\mathbf{x} \in \Omega$ is defined as: $\mathbf{x}=\left(\begin{array}{ll}x & y\end{array}\right)^{T}$ and the motion vector at location $\mathbf{x}$ and date $t \in\left[t_{0}, t_{N}\right]$ is written: $\mathbf{w}(\mathbf{x}, t)=(u(\mathbf{x}, t) \quad v(\mathbf{x}, t))^{T}$ with $u$ and $v$ belonging to $H^{2}(\Omega)$. Image observations denoted $\mathbf{Y}(\mathbf{x}, t)$ or $I(\mathbf{x}, t)$, according to the mathematical context of data assimilation or image processing, are available.

As explained in the introduction, the motion estimation method relies on image assimilation. The state vector has first to be defined. It includes the two components $u$ and $v$ of the motion vector $\mathbf{w}(\mathbf{x}, t)$ and a pseudo-image $I_{s}(\mathbf{x}, t) \in H^{2}(\Omega)$, which has similar properties than the image observations: the motion field transports the values of this pseudoimage in the same way than image pixels. The state vector is then defined as the function $\mathbf{X}(\mathbf{x}, t)=$ $\left(\mathbf{w}(\mathbf{x}, t)^{T} \quad I_{s}(\mathbf{x}, t)\right)^{T}$. Having included the pseudoimage $I_{s}$, within the state vector, allows an easy comparison to the image observations at each acquisition date: they have to be almost identical or their difference should be almost zero.

The heuristics on dynamics used in this paper is the Lagrangian constancy of velocities:

$$
\frac{d \mathbf{w}}{d t}=\frac{\partial \mathbf{w}}{\partial t}+\left(\mathbf{w}^{T} \nabla\right) \mathbf{w}=0
$$

Being generic enough, this Lagrangian constancy is a rough approximation that is usable for many image data types (Béréziat and Herlin, 2011). The pseudoimage $I_{s}$ obeys to the same heuristics than the image data: the velocity field transports it:

$$
\frac{\partial I_{s}}{\partial t}+\mathbf{w}^{T} \nabla I_{s}=0
$$

Equations (1) and (2) only being heuristics, they approximate the reality. An error term, $\varepsilon(\mathbf{x}, t)=$ $\left(\varepsilon_{\mathbf{w}}(\mathbf{x}, t)^{T} \quad \varepsilon_{I_{s}}(\mathbf{x}, t)\right)^{T}$, is added to represent this uncertainty and the evolution equation of the state vector is then summarized by:

$$
\frac{\partial \mathbf{X}}{\partial t}+\mathbb{M}(\mathbf{X})=\varepsilon
$$

As the motion value is estimated from the image observations, denoted $\mathbf{Y}(\mathbf{x}, t)$, an observation equation is required to link the state vector to these observation data:

$$
\mathbb{H} \mathbf{X}=\mathbf{Y}+\varepsilon_{R}
$$

The observation operator $\mathbb{H}$ projects the state vector into the space of observations. As the state vector includes the pseudo-image component, $\mathbb{H}$ reduces to: $\mathbb{H X}=I_{s}$. The observation error $\varepsilon_{R}(\mathbf{x}, t)$ models the acquisition noise of the image observations. 
During the motion estimation process, Eqs. (1) and (2) are integrated in time. This requests an initial value of the state vector at date $t_{0}$. Some knowledge of this value is often available and named the background $\mathbf{X}_{b}(\mathbf{x})$. However, the state vector at date $t_{0}$ is not exactly equal to that background value and an error term $\varepsilon_{B}(\mathbf{x})$ is introduced:

$$
\mathbf{X}\left(\mathbf{x}, t_{0}\right)=\mathbf{X}_{b}(\mathbf{x})+\varepsilon_{B}
$$

The error variables $\varepsilon, \varepsilon_{R}$ and $\varepsilon_{B}$ are supposed unbiased, Gaussian and characterized by their respective covariance matrices $Q, R$ and $B$.

For retrieving motion from the image observations, the equations (3), (4) and (5) must be simultaneously solved. In this paper, this is achieved with the 4D-Var method described in the next section.

\section{CONTROL OF MODEL ERROR}

The data assimilation system obtained with Eqs. (3, 5, 4) is written as:

$$
\begin{aligned}
\frac{\partial \mathbf{X}}{\partial t}+\mathbb{M}(\mathbf{X}) & =\varepsilon \\
\mathbf{X}\left(t_{0}\right) & =\mathbf{X}_{b}+\varepsilon_{B} \\
\mathbb{H} \mathbf{X} & =\mathbf{Y}+\varepsilon_{R}
\end{aligned}
$$

Looking for the solution $\mathbf{X}$ that solves System $6 \mathrm{a} / 6 \mathrm{~b} / \mathrm{cc}$ is expressed as an optimization problem. A cost function is defined that has to be minimized by controlling $\varepsilon$ and $\varepsilon_{B}$ :

$$
\begin{aligned}
J\left[\varepsilon_{B}, \varepsilon\right] & =\frac{1}{2} \int_{t}\left\langle\varepsilon(t), Q^{-1} \varepsilon(t)\right\rangle+\frac{1}{2}\left\langle\varepsilon_{B}, B^{-1} \varepsilon_{B}\right\rangle(7) \\
& +\frac{1}{2} \int_{t}\left\langle\mathbb{H} \mathbf{X}(t)-\mathbf{Y}(t), R^{-1}[\mathbb{H} \mathbf{X}(t)-\mathbf{Y}(t)]\right\rangle
\end{aligned}
$$

$\langle$,$\rangle denotes the space integral over the domain \Omega$. The first term comes from Eq. (3), the second from Eq. (5) and the third from Eq. (4). They express that the three error terms have to be minimized, accordingly to their respective covariance matrices $Q, B$ and $R$.

In order to minimize the cost function $J$, its gradient is derived with calculus of variation.

Theorem 1. The gradient of $J$ is given by:

$$
\begin{aligned}
\frac{\partial J}{\partial \varepsilon(t)}\left[\varepsilon, \varepsilon_{B}\right] & =Q^{-1} \varepsilon(t)+\lambda(t) \\
\frac{\partial J}{\partial \varepsilon_{B}}\left[\varepsilon, \varepsilon_{B}\right] & =B^{-1} \varepsilon_{B}+\lambda\left(t_{0}\right)
\end{aligned}
$$

with $\lambda(t)$ the adjoint variable computed backward in time by:

$$
\begin{aligned}
\lambda\left(t_{N}\right) & =0 \\
-\frac{\partial \lambda(t)}{\partial t}+\left(\frac{\partial \mathbb{M}}{\partial \mathbf{X}}\right)^{*} \lambda(t) & =\mathbb{H}^{T} R^{-1} \times \\
& {[\mathbb{H} \mathbf{X}(t)-\mathbf{Y}(t)] }
\end{aligned}
$$

Proof.

The state vector and the functional $J$ depend on $\varepsilon(t)$ (defined in Eq. (3p) and $\varepsilon_{B}$ (defined in Eq. (5p). Let $\delta J$ and $\delta \mathbf{X}$ be the perturbations on $J$ and $\mathbf{X}$ obtained if $\varepsilon(t)$ and $\varepsilon_{B}$ are respectively perturbed by $\delta \varepsilon(t)$ and $\delta \varepsilon_{B}$. We obtain from Eq. (7):

$$
\begin{aligned}
\delta J & =\int_{t}\left\langle\delta \varepsilon(t), Q^{-1} \varepsilon(t)\right\rangle+\left\langle\delta \varepsilon_{B}, B^{-1} \varepsilon_{B}\right\rangle \\
& +\int_{t}\left\langle\delta \mathbf{X}(t), \mathbb{H}^{T} R^{-1}[\mathbb{H} \mathbf{X}(t)-Y(t)]\right\rangle
\end{aligned}
$$

and from Eqs. (3) and (5):

$$
\begin{aligned}
\frac{\partial \delta \mathbf{X}(t)}{\partial t}+\frac{\partial \mathrm{M}}{\partial \mathbf{X}} \delta \mathbf{X}(t) & =\delta \varepsilon(t) \\
\delta \mathbf{X}\left(t_{0}\right) & =\delta \varepsilon_{B}
\end{aligned}
$$

Eq. 10a gives, after multiplication by $\lambda(t)$ and integration on the space-time domain, the following equality:

$\int_{t}\left\langle\frac{\partial \delta \mathbf{X}(t)}{\partial t}, \lambda(t)\right\rangle+\int_{t}\left\langle\frac{\partial \mathbf{M}}{\partial \mathbf{X}} \delta \mathbf{X}(t), \lambda(t)\right\rangle=\int_{t}\langle\delta \varepsilon(t), \lambda(t)\rangle$

Integration by parts is applied on the first term and the adjoint operator is used in the second one in order to obtain:

$$
\begin{array}{r}
-\int_{t}\left\langle\delta \mathbf{X}(t), \frac{\partial \lambda(t)}{\partial t}\right\rangle+<\delta \mathbf{X}\left(t_{N}\right), \lambda\left(t_{N}\right)> \\
-<\delta \varepsilon_{B}, \lambda\left(t_{0}\right)>+\int_{t}\left\langle\delta \mathbf{X}(t), \frac{\partial \mathbb{M}^{*}}{\partial \mathbf{X}} \lambda(t)\right\rangle \\
=\int_{t}\langle\delta \varepsilon(t), \lambda(t)\rangle
\end{array}
$$

From Eq. $8 \mathrm{a}$, it comes that $<\delta \mathbf{X}\left(t_{N}\right), \lambda\left(t_{N}\right)>$ has a null value. Eq. $8 \mathrm{~b}$ is then used to obtain:

$$
\begin{array}{r}
-\left\langle\delta \mathbf{X}(t), \frac{\partial \lambda(t)}{\partial t}\right\rangle+\left\langle\delta \mathbf{X}(t), \frac{\partial \mathbb{M}^{*}}{\partial \mathbf{X}} \lambda(t)\right\rangle= \\
<\delta \mathbf{X}(t), \mathbb{H}^{T} R^{-1}[\mathbb{H} \mathbf{X}(t)-Y(t)]>
\end{array}
$$

and Eq. (11) is rewritten as:

$$
\begin{array}{r}
\int_{t}<\delta \mathbf{X}(t), \mathbb{H}^{T} R^{-1}[\mathbb{H} \mathbf{X}(t)-Y(t)]>= \\
<\delta \varepsilon_{B}, \lambda\left(t_{0}\right)>+\int_{t}\langle\delta \varepsilon(t), \lambda(t)\rangle
\end{array}
$$

From this and Eq. (9), we derive:

$$
\begin{aligned}
\delta J & =\int_{t}<\delta \varepsilon(t), Q^{-1} \varepsilon(t)>+<\delta \varepsilon_{B}, B^{-1} \varepsilon_{B}> \\
& +\quad<\delta \varepsilon_{B}, \lambda\left(t_{0}\right)>+\int_{t}\langle\delta \varepsilon(t), \lambda(t)\rangle
\end{aligned}
$$

and obtain the gradient of $J$ as given in Theorem 1 .

$$
\begin{aligned}
\frac{\partial J}{\partial \varepsilon(t)}\left[\varepsilon(t), \varepsilon_{B}\right] & =Q^{-1} \varepsilon(t)+\lambda(t) \\
\frac{\partial J}{\partial \varepsilon_{B}}\left[\varepsilon(t), \varepsilon_{B}\right] & =B^{-1} \varepsilon_{B}+\lambda\left(t_{0}\right)
\end{aligned}
$$


The cost function $J$ is minimized using an iterative steepest descent method. At each iteration, the forward time integration of $X$ is performed and provides $J$, then a backward integration of $\lambda$ computes $\nabla J$. An efficient solver (Byrd et al., 1995), based on the BFGS method, is used to perform the steepest descent given $J$ and $\nabla J$.

\section{NUMERICAL IMPLEMENTATION}

An explicit Euler scheme is applied to perform the forward time integration of $X$. Time indexes go from 0 to $N_{t}$ for the assimilation window $\left[t_{0}, t_{N}\right]$. Moreover a robust numerical scheme is proposed to discretize in space. Eq. (1) is replaced by a system of two equations respectively describing the evolution of components $u$ and $v$ of the velocity:

$$
\begin{aligned}
& \frac{\partial u}{\partial t}+u \frac{\partial u}{\partial x}+v \frac{\partial u}{\partial y}=0 \\
& \frac{\partial v}{\partial t}+u \frac{\partial v}{\partial x}+v \frac{\partial v}{\partial y}=0
\end{aligned}
$$

Each equation contains a linear advection part and a non linear one. A source splitting, (Wolke and Knoth, 2000), is first applied on each equation and illustrated on Eq. (12). Given an interval $\left[t_{1}, t_{2}\right]$, the two following equations are integrated independently:

$$
\begin{aligned}
& \frac{\partial u^{*}}{\partial t}+u^{*} \frac{\partial u^{*}}{\partial x}=0 \quad t \in\left[t_{1}, t_{2}\right] \\
& \frac{\partial u^{* *}}{\partial t}+v \frac{\partial u^{* *}}{\partial y}=0 \quad t \in\left[t_{1}, t_{2}\right]
\end{aligned}
$$

with $u^{*}\left(x, y, t_{1}\right)=u^{* *}\left(x, y, t_{1}\right)=u\left(x, y, t_{1}\right) . \quad u\left(x, y, t_{2}\right)$ is then approximated as $u\left(x, y, t_{2}\right)=u^{* *}\left(x, y, t_{2}\right)+$ $\left(u^{*}\left(x, y, t_{2}\right)-u^{*}\left(x, y, t_{1}\right)\right)$.

The linear advection of Eq. (15) is approximated by a first order up-wind scheme, (Hundsdorfer and Spee, 1995). The non linear advection of Eq. (14) is first rewritten in a conservative form, $\frac{\partial u}{\partial t}+\frac{\partial}{\partial x}\left(\frac{1}{2} u^{2}\right)=0$, and approximated by a first order Godunov scheme, (LeVeque, 1992).

The backward time integration of the adjoint variable involves the adjoint operator $\left(\frac{\partial \mathrm{M}}{\partial X}\right)^{*}$ (Eq. $8 \mathrm{~b}$ ) that is obtained thanks to an automatic differentiation software, (Hascoët and Pascual, 2004).

\section{EXPERIMENTS AND RESULTS}

Two algorithms have been implemented and compared during the experimental phase in order to demonstrate the improvement provided by the additional error term in the evolution equation. The first one, named IM for Imperfect Model, relies on Eqs. 345 with the error term $\varepsilon(t)$ included in the evolution equation of the state vector. The second one, named PM for Perfect Model, satisfies the same equations, but the error term is suppressed in Eq. (3). The first term of the cost function $J$ in Eq (7) vanishes for PM and minimization is then only controlled by $\varepsilon_{B}$.

We designed a series of twin experiments for assessing the impact of the error term $\varepsilon(t)$. Three of them are described in the following.

Given initial conditions, at index 0 , on velocity and pseudo-image $\left(\mathbf{w}_{\text {ref }}(0), I_{S}(0)\right)^{T}$, as displayed in Figure 1, and given a model error $\varepsilon_{\text {ref }}$, Eq. (3) is integrated in time. We consider the snapshots of the

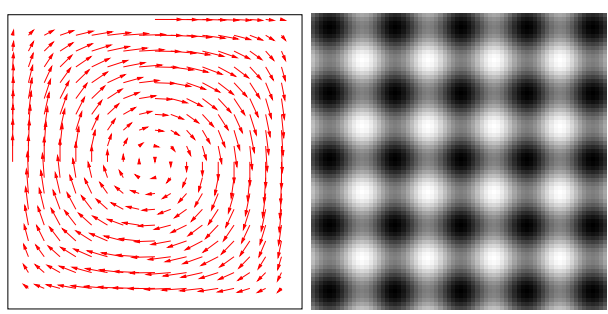

Figure 1: initial conditions of the velocity field and pseudoimage.

pseudo-image function, obtained by the simulation at given indexes $k_{i}$, to define the image observations used in the assimilation experiments. In that way, we have full knowledge of the initial motion field $\mathbf{w}_{\text {ref }}(0)$ and of the error values $\varepsilon_{\text {ref }}(t)$ that produce the observations and we can quantitatively compare them with the results of the assimilation method obtained by IM and PM.

The background value, $\mathbf{X}_{b}=\left(\mathbf{w}_{b}, I_{s b}\right)^{T}$, in Eq. 5), is the same for IM and PM. The pseudo-image $I_{s b}(\mathbf{x})$ is chosen as the first observation denoted $\mathbf{Y}\left(\mathbf{x}, k_{1}\right)$. For these experiments, we choose $\mathbf{w}_{b}(\mathbf{x})=\overrightarrow{0}$. As we do not want to constrain $\mathbf{w}(0)$ to stay close to $\overrightarrow{0}$ during the optimization process, the background term of $J$, in Eq. 77, reduces to $\left\langle\varepsilon_{B_{I_{s}}}, B_{I_{s}}^{-1} \varepsilon_{B_{I_{s}}}\right\rangle$. We empirically set $B_{I_{s}}$ and $R$ to 1 for IM and PM. For IM, $Q$ is a $2 \times 2$ diagonal matrix whose coefficients, $Q_{u}$ and $Q_{v}$ have small values. We use five image observations. The discrete assimilation window has indexes from 0 to $N_{t}=83$ and observation images are available at indexes $k_{1}$ to $k_{5}=1,21,41,61$ and 81 .

Experiment 1. We first assess the results of IM in the case where the error value $\varepsilon(t) \equiv \overrightarrow{0}$ during the simulation that creates the observation images. Questions are the following. IM being designed to estimate an error value, will it be able to correctly estimate that 


\begin{tabular}{c|cc|cc} 
& \multicolumn{2}{|c|}{$\left|\theta-\theta_{\text {ref }}\right|$} & \multicolumn{2}{|c}{$\left\|\mathbf{w}-\mathbf{w}_{\text {ref }}\right\| /\left\|w_{\text {ref }}\right\|$} \\
Method & mean & st. dev. & mean & st. dev. \\
\hline \hline PM & 0.82 & 2.24 & 0.018 & 0.046 \\
IM & 0.79 & 2.11 & 0.023 & 0.046
\end{tabular}

Table 1: First Experiment. Statistics on the inaccuracy of the motion fields estimated by PM and IM at index 0 .

error with a null value? Being designed with no error term, will PM obtain a better estimation of the real motion field $\mathbf{w}_{\text {ref }}(0)$ than IM?

The first observation is the initial condition of Figure 1 and the four next one are displayed on Figure 2

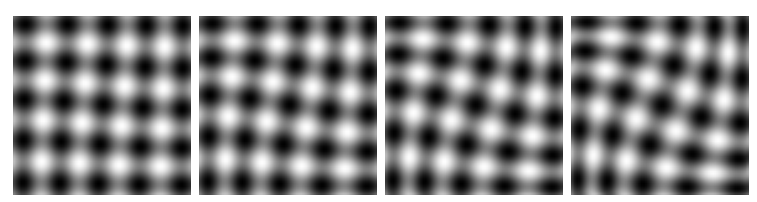

Figure 2: First Experiment. Observation Images.

A qualitative and quantitative comparison of the initial motion fields retrieved by $\mathrm{PM}, \mathbf{w}^{\mathrm{PM}}(0)$, and $\mathrm{IM}, \mathbf{w}^{\mathrm{IM}}(0)$, with the ground truth, $\mathbf{w}_{\text {ref }}(0)$, is achieved. Figure 3 displays these three velocity fields. They are visually identical: both PM and IM correctly estimate motion. To quantitatively compare a

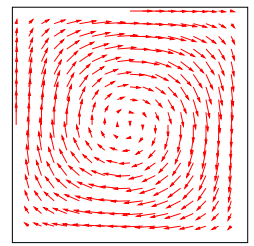

(a) $\mathbf{w}_{\text {ref }}(0)$

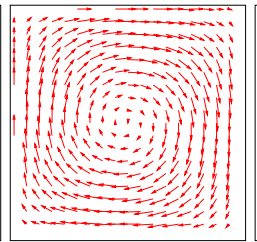

(b) $\mathbf{w}^{\mathrm{PM}}(0)$

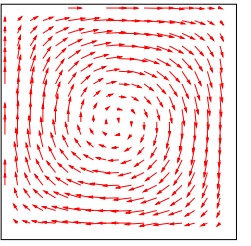

(c) $\mathbf{w}^{\mathrm{IM}}(0)$
Figure 3: First Experiment. Comparison of the estimations with ground truth.

velocity result $\mathbf{w}$ with the ground truth $\mathbf{w}_{\text {ref }}$ at index 0 , average and standard deviation of the absolute angular error $\left|\theta-\theta_{\text {ref }}\right|$ and the relative norm difference $\left(\left\|\mathbf{w}-\mathbf{w}_{\text {ref }}\right\| /\left\|w_{\text {ref }}\right\|\right)$ are measured. These values are provided in Table 1 for PM and IM. This confirms that the two methods compute a correct velocity field with a mean angular inaccuracy less than 1 degree and an average of the relative norm difference around $2 \%$. In conclusion, the presence of the error term $\varepsilon(t)$ has no negative impact if the observation data have been produced without adding error in the evolution equation during the simulation process.

Experiment 2. In that experiment, the error term, $\varepsilon_{\text {ref }}(t)$, used during the simulation of the image observations, has a constant value over the space-time domain. To study separately the errors on the dynamics (motion components) and on the pseudo-image, we constrain the temporal evolution of the pseudo-image to be perfect and exactly satisfy the transport equation. We set $\varepsilon_{\mathrm{ref}}(t)(\mathbf{x})=\left(\begin{array}{lll}10^{-3} & 10^{-3} & 0\end{array}\right)^{T}$. The value $10^{-3}$ corresponds to a final cumulative error of $70 \%$ of the maximum of the initial velocity norm.

The first observation is the initial condition (Figure 1) and the four next ones are obtained by integrating Eq. (3) (see Figure 4).

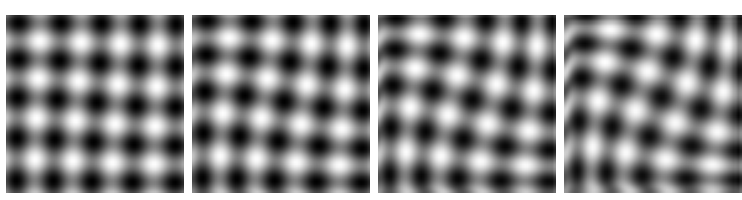

Figure 4: Second Experiment. Observations Images.

Results are displayed on Figure 5. As it can be seen, only IM computes a correct velocity field while PM completely fails due to the noise included during the simulation that produces the observations. PM, relying on a perfect evolution equation, is unable to retrieve the correct solution and computes the initial velocity field that is the best compromise between the evolution model and the observations. Table 2

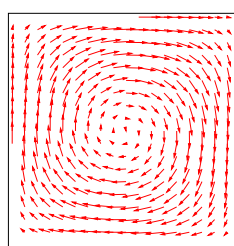

(a) $\mathbf{w}_{\text {ref }}(0)$

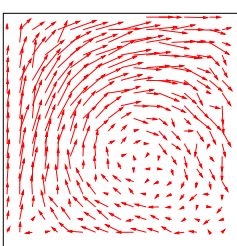

(b) $\mathbf{w}^{\mathrm{PM}}(0)$

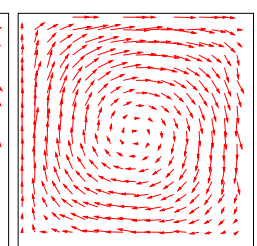

(c) $\mathbf{w}^{\mathrm{IM}}(0)$
Figure 5: Second Experiment. Comparison of the estimations with ground truth.

provides statistics on the difference between the motion results and the ground truth: it confirms that IM significantly improves the motion estimation both in norm and in direction, due to the error term added in the evolution equation.

\begin{tabular}{c|cc|cc} 
& \multicolumn{2}{|c|}{$\left|\theta-\theta_{\text {ref }}\right|$} & \multicolumn{2}{c}{$\left\|\mathbf{w}-\mathbf{w}_{\text {ref }}\right\| /\left\|w_{\text {ref }}\right\|$} \\
Method & mean & st. dev. & mean & st. dev. \\
\hline \hline PM & 24.16 & 30.34 & 0.32 & 0.44 \\
IM & 5.98 & 11.40 & 0.11 & 016
\end{tabular}

Table 2: Second Experiment. Statistics on the inaccuracy of the motion fields estimated by PM and IM at index 0 .

Experiment 3. In that experiment, $\varepsilon_{\text {ref }}(t)$, used during the simulation of the image observations, is constant in space and random in time. We consider a Gaussian noise with a variance of $10^{-5}$ for both components $u$ and $v$.

The first observation is the initial condition of Figure 1 and the four next one are displayed on Figure 6 


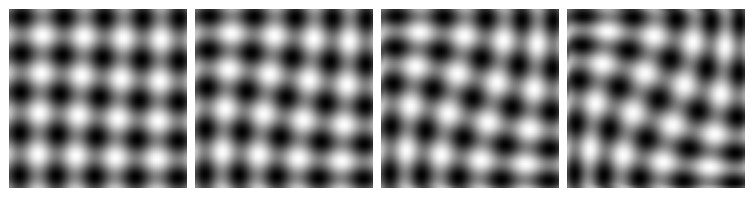

Figure 6: Third Experiment. Observations Images.

As in the first experiment, the initial motion fields retrieved by PM and IM are qualitatively similar to the ground truth. Table 3 gives statistics on the difference between the results and the ground truth. It shows that both methods estimate correctly the velocity with a slight advantage to IM.

\begin{tabular}{c|cc|cc} 
& \multicolumn{2}{|c|}{$\left|\theta-\theta_{\text {ref }}\right|$} & \multicolumn{2}{c}{$\left\|\mathbf{w}-\mathbf{w}_{\text {ref }}\right\| /\left\|w_{\text {ref }}\right\|$} \\
Method & mean & st. dev. & mean & st. dev. \\
\hline \hline PM & 6.91 & 10.14 & 0.13 & 0.57 \\
IM & 5.34 & 8.09 & 0.10 & 0.48
\end{tabular}

Table 3: Third Experiment. Statistics on the inaccuracy of the motion fields estimated by PM and IM at index 0 .

\section{CONCLUSIONS}

This paper discusses a data assimilation method that simultaneously estimates motion from image data and the inaccuracy in the evolution equation used to model the dynamics of that motion field. For that purpose, an error term is added to the evolution equation, which is part of the data assimilation system, and controlled by the optimization method. As a result, the method provides the motion field and the error value on the dynamics at each time step of the assimilation window.

The method, named IM as Imperfect Model, has been quantified on twin experiments and compared with a Perfect Model, named PM, that does not involve the error term. All experiments showed that IM better estimates motion if the dynamics is not accurately described by the evolution equation: an error term has been added during the synthesis of image observations. The improvement obtained with IM is clearly visible in the second experiment that presents a large deviation of the real dynamics to the evolution equation. In that case, the perfect model PM completely fails to retrieve the motion field. This is clearly visible when motion results are displayed. In all other experiments, a quantitative improvement is obtained with the Imperfect Model, if the simulation creating the image observations included some error.

An important perspective of that research work would be, for instance, the detection of changes of dynamics over long temporal sequences.

\section{REFERENCES}

Béréziat, D. and Herlin, I. (2011). Solving ill-posed image processing problems using data assimilation. Numerical Algorithms, 56(2):219-252.

Béréziat, D., Herlin, I., and Younes, L. (2000). A generalized optical flow constraint and its physical interpretation. In $C V P R$, pages 487-492.

Byrd, R. H., Lu, P., and Nocedal, J. (1995). A limited memory algorithm for bound constrained optimization. Journal on Scientific and Statistical Computing, 16(5):1190-1208.

Hascoët, L. and Pascual, V. (2004). Tapenade 2.1 user's guide. Technical Report 0300, INRIA.

Horn, B. and Schunk, B. (1981). Determining optical flow. Artificial Intelligence, 17:185-203.

Hundsdorfer, W. and Spee, E. (1995). An efficient horizontal advection scheme for the modeling of global transport of constituents. Monthly Weather Review, 123(12):3,554-3,564.

Isambert, T., Berroir, J., and Herlin, I. (2008). A multiscale vector spline method for estimating the fluids motion on satellite images. In ECCV, pages 665-676. Springer.

Le Dimet, F.-X. and Talagrand, O. (1986). Variational algorithms for analysis and assimilation of meteorological observations: Theoretical aspects. Tellus, 38A:97110.

LeVeque, R. (1992). Numerical Methods for Conservative Laws, chapter Godunov's method. Lectures in Mathematics. ETH Zürich, Birkhaüser Verlag, 2nd edition.

Papadakis, N., Corpetti, T., and Mémin, E. (2007). Dynamically consistent optical flow estimation. In ICCV, pages $1-7$.

Papadakis, N., Mémin, E., and Cao, F. (2005). A variational approach for object contour tracking. In ICCV workshop on variational, geometric and level set methods in computer vision, pages 259-270.

Tikhonov, A. N. (1963). Regularization of incorrectly posed problems. Soviet mathematics - Doklady, 4:16241627.

Titaud, O., Vidard, A., Souopgui, I., and Le Dimet, F.-X. (2010). Assimilation of image sequences in numerical models. Tellus A, 62:30-47.

Trémolet, Y. (2006). Accounting for an imperfect model in 4D-Var. Quaterly Journal of the Royal Meteorological Society, 132(621):2483-2504

Valur Hólm, E. (2008). Lectures notes on assimilation algorithms. European Centre for Medium-Range Weather Forecasts Reading.

Wolke, R. and Knoth, O. (2000). Implicit-explicit RungeKutta methods applied to atmospheric chemistrytransport modelling. Environmental Modelling and Software, 15:711-719. 